\title{
Tensiones entre los saberes académicos y los movimientos sociales en las problemáticas ambientales*
}

Conflict between Academic Knowledge and Social Movements within Environmental

Issues

\author{
Florencia Arancibia ${ }^{\dagger}$ \\ Ignacio Bocles ${ }^{\ddagger}$ \\ Alicia Massarini ${ }^{\S}$ \\ Damián Verzeñassi ${ }^{\dagger \dagger}$
}

\begin{abstract}
Resumen
Abordamos la reflexión sobre la tensión que se establece entre el saber académico, las problemáticas ambientales y la experiencia de las comunidades afectadas, en el conflicto dado por la expansión del modelo agrario basado en la producción de organismos genéticamente modificados (OGM) en Argentina. Mientras sectores académicos hegemónicos promovieron activamente la expansión de esta tecnología, se multiplicaron las denuncias sobre la incidencia de graves problemas de salud en poblaciones rurales expuestas a los agroquímicos que este modelo utiliza masivamente. Estas denuncias fueron generalmente ignoradas por los primeros, por los medios de comunicación y por los organismos responsables de velar por la salud pública. Ante ello, las comunidades afectadas se han enfrentado a la necesidad de hacer público su problema sanitario. Una estrategia explorada fue la de buscar apoyo en la Academia, encontrando eco en unos pocos científicos cuyos estudios pusieron de manifiesto la relación entre ciertos agroquímicos y los daños denunciados. Sin embargo, al asumir la lógica y la metodología reduccionista de los saberes científicos esta estrategia enfrentó serias limitaciones. En este contexto, en 2015 se conformó el Grupo de Epidemiología de la Ciencia Digna, que buscó desarrollar nuevas estrategias de investigación-acción para coproducir conocimiento entre científicos y comunidades, intentando superar las limitaciones mencionadas. Retomando conceptos y debates de la filosofía de la ciencia, en este artículo pretendemos reflexionar sobre estas nuevas estrategias, así como aportar ideas e interrogantes para contribuir a su desarrollo.
\end{abstract}

Palabras clave: conflictos socio-ambientales - biotecnología - agroquímicos - movimientos sociales - epistemología crítica

\begin{abstract}
In this paper we address some tensions among academic knowledge, environmental issues and communities' experience, within the conflict over the production of genetically modified organisms (GMOs) in Argentina. While hegemonic groups within academia actively promoted the expansion of this technology, a growing number of rural communities exposed to pesticides reported serious health problems. These claims were generally ignored by the hegemonic groups within academia, as well as by the media and public health agencies. In this way, communities faced the need to make their health problems public. Seeking help within Academia was one of the most common strategies explored by communities. This triggered the support of a few sympathetic scientists who pursued studies proving association between certain pesticides and the reported illnesses. However, this strategy faced serious problems, since it was mainly based on a scientific-reductionist logic. The Group of Worthy Science was created in 2015, aiming to develop new research strategies to co-produce knowledge between scientists and communities and overcome these barriers. Drawing upon concepts of the philosophy of science, our goal is to reflect upon these new strategies, as well as suggest new questions and ideas to contribute to their further development.
\end{abstract}

Keywords: socio-environmental conflicts - biotechnology - pesticides - social movements - critical epistemology

* Recibido: 31 de Marzo de 2016. Aceptado con revisiones: 5 de Diciembre de 2016.

† Grupo de Epidemiología de la Ciencia Digna/Becaria Pos-Doctoral de CONICET en CENIT, Universidad Tres de Febrero. Para contactar al autor, por favor, escribir a: florenciapaulaarancibia@gmail.com.

* Grupo de Epidemiología de la Ciencia Digna/Facultad de Medicina, niversidad de Buenos Aires. Para contactar al autor, por favor, escribir a: i.bocles@gmail.com.

$\S$ Grupo de Epidemiología de la Ciencia Digna/CONICET/Universidad de Buenos Aires. Para contactar al autor, por favor, escribir a: aliciamassarini@yahoo.com.ar.

${ }^{+\dagger}$ Grupo de Epidemiología de la Ciencia Digna/Instituto de Salud Socioambiental, Facultad de Ciencias Médicas, Universidad Nacional de Rosario. Para contactar al autor, por favor, escribir a: damianverze@yahoo.com.ar.

Metatheoria 8(2)(2018): 105-123. ISSN 1853-2322.

(c) Editorial de la Universidad Nacional de Tres de Febrero. Publicado en la República Argentina. 


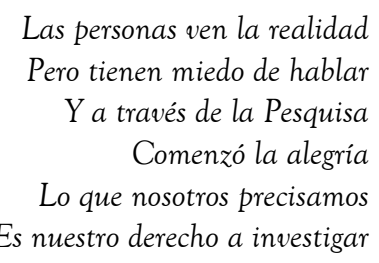

“A pesquisa”, poesía de un labrador de Goaias escrita durante una experiencia de investigación participativa en la zona rural brasileña en 1980 .

\section{Introducción}

En Argentina, a partir de la aprobación de la soja transgénica resistente al glifosato (RR) en 1996, la superficie sembrada con cultivos transgénicos no ha cesado de crecer, abarcando en la actualidad 24,5 millones de hectáreas. ${ }^{1}$ En ausencia de debate social, el modelo de producción industrial de la agrobiotecnología se expandió rápidamente, desplazando otros cultivos destinados al autoconsumo, otras producciones agropecuarias -como la ganadería, los tambos, la fruticultura-, produciendo un cambio notable en los agroecosistemas y especialmente en las relaciones sociales en el campo. La desaparición de centenas de pueblos rurales, de establecimientos productivos pequeños y medianos, el éxodo y desempleo rural, la mayor concentración en la tenencia de la tierra, son algunas de las secuelas de este modelo de agricultura sin agricultores (Giarraca 2006, Teubal 2005, Teubal \& Giarracca 2005, Teubal 2008). Al mismo tiempo el incremento exponencial en el uso de agroquímicos que acompañó esta expansión ha provocado importantes impactos ambientales y en la salud de las poblaciones de áreas rurales, que concentran más de 12 millones de habitantes.

Frente a estos cambios, desde hace más de una década, diversas comunidades han comenzado a dar cuenta de los impactos ambientales y los daños en la salud que están experimentando. Generalmente, tanto los medios de comunicación como los organismos gubernamentales han ignorado o silenciado estos reclamos. Por su parte, en el ámbito académico se advierte una clara polarización, acompañada de una notable asimetría: mientras la mayor parte de las voces de especialistas y académicos han apoyado e incluso promovido activamente el modelo de la agrobiotecnología con transgénicos, un pequeño grupo de científicos -en muchos casos aislados y hostigados en sus instituciones- se ha hecho cargo de investigar y dar a conocer los daños y los impactos negativos que produce. En este escenario se instala la necesidad de caracterizar los conflictos y tensiones que se establecen en el ámbito científico y, particularmente, analizar las relaciones que se establecen entre el conocimiento académico y las demandas de las comunidades, con el propósito de explorar y promover alterativas que contribuyan a un cambio.

Este artículo se organiza en 6 secciones. En la introducción hemos descrito brevemente las transformaciones e impactos generados a partir de la expansión del cultivo de OGMs en Argentina y en virtud de ello, la problemática que se genera en relación a las demandas de las comunidades afectadas y en el seno de la comunidad científica; en la segunda sección, "La perspectiva teóricoanalítica”, en que se presentan las principales nociones y concepciones que enmarcan la propuesta que aquí expondremos, comprende tres aspectos: en el apartado 2.1 argumentamos acerca de la pertinencia de la reflexión filosófica sobre la problemática abordada; en el apartado 2.2 exponemos y problematizamos algunas concepciones acerca de las relaciones entre ciencia, tecnología y sociedad, su dimensión política y ética; y en el apartado 2.3 proponemos algunas ideas que podrían contribuir a la construcción de alternativas para la producción de un conocimiento "situado y transformador". La tercera sección, "Reflexiones sobre el estado actual de los saberes académicos y resistencias sociales relativas a la expansión del modelo de la agrobiotecnología", comprende dos apartados: 3.1 en que caracterizamos la actual etapa de la tecno-ciencia, sus impactos en nuestra región y los principales ejes

\footnotetext{
${ }^{1}$ Esta superficie representa aproximadamente el $14 \%$ de la superficie global, Argentina es el tercer productor mundial de este tipo de cultivos, después de Estados Unidos y Brasil. Fuente: http://www.argenbio.org/index.php?action=cultivos\&opt=5.
} 
del discurso hegemónico de científicos y funcionarios que apuntan a legitimar el actual modelo productivo de la agrobiotecnología; y 3.2. dónde reseñamos las principales experiencias de resistencia tanto en el ámbito científico como en el de los territorios. En la cuarta sección "La propuesta del Grupo de Epidemiología de la Ciencia Digna” exponemos los criterios y las características de la propuesta que está siendo construida por el grupo al que pertenecemos, en la quinta sección reseñamos las principales conclusiones que se desprenden de este trabajo y finalmente en la sección 6 "Preguntas y propuestas abiertas por el proyecto", dejamos planteados algunos de los principales interrogantes y aspectos aun no resueltos, que podrían dar lugar a nuevos debates que consideramos indispensables en este proceso de construcción colectiva.

\section{Perspectiva teórico-analítica}

\section{1. ¿Por qué acudir a la filosofía para pensar este problema?}

Pigliucci (2008) enumera las distintas líneas de investigación de la filosofía de la ciencia y destaca que una de ellas se focaliza en la relación entre ciencia y sociedad:

Aquí el filósofo se convierte en un crítico no sólo de cómo se están llevando a cabo la tarea científica y la interpretación de sus hallazgos, sino principalmente, de cómo estos hallazgos son entendidos por el público y cómo se los utiliza para orientar las políticas sociales (Kitcher 2001, tomado de Pigliucci 2008, p. 10)

Esta línea de investigación filosófica se desenvuelve en una región de frontera a la que Pigliucci denomina "sci-phi", interface que se torna terreno fértil para el tipo de interdisciplinariedad que puede mover ambos campos, produciendo un efecto positivo tanto en los estudiantes y científicos como en el público en general. Sin embargo, los científicos han sido hasta ahora más bien reticentes a considerar el valor del análisis filosófico para su disciplina (Pigliucci 2008). Contrariamente a esta tendencia, en este trabajo partimos de la idea que la reflexión filosófica es particularmente relevante a la hora de abordar problemas sociales complejos en las que interviene la ciencia y particularmente aquellas problemáticas socio-ambientales que se establecen como resultado de los impactos de la tecno-ciencia.

Nuestro objetivo es analizar la tensión que se establece entre el saber académico, la experiencia de las comunidades y las problemáticas ambientales en el conflicto por la expansión del modelo agrario basado en el cultivo extensivo de organismos genéticamente modificados (OGM) destinados a la exportación en Argentina. Reflexionamos sobre estas tensiones a través de conceptos y debates de la filosofía de la ciencia, jerarquizando las dimensiones política, ideológica y ética involucradas, con la intención de colaborar en el desarrollo de nuevas estrategias de investigación-acción que actualmente están siendo desarrolladas y experimentadas por poblaciones afectadas y científicos comprometidos con ellas.

\subsection{La acción política en el escenario de la tecno-ciencia: enfrentando un falso dilema}

Según Moore et al. (2011) en un sistema capitalista global donde la acumulación de capital es motorizada por avances de la tecnociencia, las políticas que regulan las nuevas tecnologías cada vez más basan su legitimidad en un discurso tecno-científico -lo que ya Habermas (1970) denominaba como un proceso de "cientifización" de la política-. En este contexto, según los autores, los movimientos y organizaciones sociales que quieran cuestionar y cambiar las políticas públicas se enfrentan con un dilema, deberán elegir entre: a) mantenerse en los márgenes del debate, cuestionando el hecho de que las decisiones políticas se deban sustentar en bases tecno-científicas y proponer otras bases centradas en el debate político e ideológico; y b) "seguir las reglas establecidas" y entrar en el juego, es decir adquirir la experticia necesaria o aliarse con expertos/científicos para producir datos científicos alternativos que pongan en duda las decisiones políticas tomadas. Según la interpretación de Moore et al. (2011) en el primer caso, se abogaría contra la "cientifización" de la política, lo cual tal vez pueda resultar positivo en un futuro, pero probablemente implique que dichos movimientos queden afuera de los procesos de 
toma de decisiones político-estatales específicos en el presente. En el segundo caso, probablemente se adquiriría mayor capacidad de influir en las decisiones político-estatales presentes, pero se correría el riesgo de seguir reproduciendo la lógica de la "cientifización" de la política.

Consideramos que si bien las reflexiones de Moore et al. dan cuenta del difícil escenario que deben enfrentar algunos movimientos sociales en la actualidad, las opciones de acción presentadas en función del dilema planteado no son exhaustivas, y que las estrategias posibles no se agotan en las dos alternativas enunciadas, lo cual sugiere que estaríamos frente a un "falso dilema". Consideramos que al menos en parte, las limitaciones de la visión de Moore et al. se deben a que su caracterización del problema asume implícitamente la noción clásica de "neutralidad de la ciencia", estableciendo una frontera infranqueable entre tecno-ciencia y política (como si los debates científicos no pudieran ser políticos y viceversa). Así, el dilema termina por reproducir la noción hegemónica que intenta criticar, legitimando el papel jerarquizado de la tecno-ciencia. Esto no significa que no existan o hayan existido movimientos que reproduzcan la lógica cientificista -lo que Moore et al. (2011) plantean como segunda opción-, pero consideramos que existe una tercera opción "superadora" que se basa en otra concepción (no hegemónica) sobre el papel de la tecno-ciencia. Es justamente esta tercera opción, basada en una mirada alternativa y crítica que parte de afirmar la "no neutralidad" de la ciencia y la tecnología y desestima el papel de "árbitro" que se atribuye al conocimiento experto en los conflictos socioambientales, la que sustenta la estrategia que planteamos desde el grupo "Epidemiología de la Ciencia Digna". Por ello antes de avanzar, creemos importante retomar un debate histórico en la filosofía y la sociología de la ciencia sobre la relación entre ciencia y política, o ciencia y sociedad, que subyace al tema de las estrategias.

Las visiones hegemónicas sobre la ciencia plantean una fuerte dicotomía entre el conocimiento científico y el conocimiento "lego". Esta dicotomía se basa en el modelo de "déficit cognitivo" (Miller, 2001, Lewenstein 2002) según el cual, la comunidad científica siempre sabe "más y mejor" y el público que no pertenece a dicha comunidad se percibe como una audiencia pasiva con déficit de conocimientos. En esta concepción, el conocimiento científico se jerarquiza sobre cualquier otra lógica, y dado que se lo asume como neutral y objetivo, los únicos problemas que se presentan en la relación ciencia-sociedad son aquellos que surgen cuando el público no está dispuesto a seguir las prescripciones de los expertos o los científicos, debido a su propia ignorancia. En consecuencia, según este modelo, es de esperar que, a mayor comprensión pública de las verdades científicas y desarrollos tecnológicos, mayor será la aceptación pública de los avances científicos y de la innovación tecnológica. En la misma dirección, Folguera señala que la forma hegemónica de entender el vínculo que se establece entre las problemáticas sociales y los conocimientos científicos siempre es asimétrico y jerárquico.

[c]uando estas problemáticas involucran la ciencia y la tecnología prevalece la idea de que éstas pueden ser comprendidas y resueltas racionalmente mediante el abordaje exclusivo de las ciencias naturales y sociales, poniendo a las ciencias en el lugar de autoridad y otorgándoles la palabra "autorizada" para la legitimación de ciertos discursos [...] (Massarini \& Schnek 2015, p. 37).

\subsection{Transitando alternativas superadoras}

Esta visión simplista sobre la relación ciencia-sociedad o ciencia-política ha sido cuestionada por diversos autores dentro de la filosofía y de la sociología de la ciencia. Uno de los autores que asumen una postura crítica es Wynne (2001), quien plantea que la opinión predominante de la relación entre ciencia y sociedad se basa en una idea no-problematizada del conocimiento científico, como si éste se tratara simplemente de "proposiciones con pretensiones de verdad" (propositional truth claims). Es decir, se omite el hecho de que los conocimientos científicos también encarnan valores culturales y compromisos éticos. De este modo, se reproduce y legitima una dicotomía extrema entre experticia y conocimiento lego:

Los enfoques convencionales [...] reproducen la histórica división categórica entre, conocimiento objetivo y real, por un lado, y emociones o valores cognitivamente vacíos, por otro; y mientras la ciencia 
se ocupa de los primeros, los segundos sólo son capaces de tomar posiciones que se consideran sentimentales, emocionales e intelectualmente vacías (Wynne 2001, p. 445).

La preocupación de distintos grupos sociales sobre impactos no previstos o no anunciados de la introducción de nuevas tecnologías (como OGMs) ha sido interpretada por diversos autores como una crisis de confianza pública en la ciencia: como si la gente no experta estuviera perdiendo el sentido de confianza en la ciencia y en la experticia. Desde el modelo de déficit, dicha crisis fue atribuida a la "ignorancia" del público. Sin embargo, Wynne plantea otro argumento. Propone que en realidad las actitudes escépticas del público no son reacciones a los riesgos en sí mismos (supuestamente erróneamente percibidos), sino que reflejan opiniones sobre las instituciones científicas y políticas, y sobre su accionar (Wynne 1980, 1989).

Por otra parte, según Wynne, la ciencia utilizada en contextos públicos tiene que lidiar con tipos de incertidumbre mucho más radicales de los que son admitidos por la misma ciencia. Además de las incertidumbres conocidas, existen la ignorancia ${ }^{2}$ y la indeterminación. ${ }^{3}$ Así, el descontento público sobre la visión de los expertos se basaría en la conciencia y el reconocimiento (posiblemente implícito) de las deficiencias y limitaciones de los conocimientos científicos por parte de los "no expertos". Sin embargo, las instituciones científicas y políticas dominantes conciben al conocimiento lego como epistemológicamente vacuo, como si no tuviese nada que "ofrecer" a los conocimientos científicos, más allá de algunas preocupaciones éticas o sentimentales. Al público se le brindan sólo dos opciones: reconocer y aceptar lo que dicen los científicos sobre los riesgos, o -si rechazan y cuestionan esto- ser catalogados como irracionales y emotivos. No se concibe la idea de que el público pueda tener marcos de sentidos diferentes y autónomos respecto de aquellos planteados por las instituciones científicas. Wynne plantea que ésta es una visión limitada de la relación entre la ciencia y el conocimiento "no experto", "que no reconoce nada de la fluidez, la porosidad y la constructividad de los límites que se establecen entre ambos" (Wynne 2001, p. 62). Y, lo más significativo, es que esta visión termina por proteger a las instituciones científicas de cualquier tipo de visión crítica relativa a sus limitaciones implícitas, valores y prejuicios culturales sobre los cuales se constituyen (los cuales no son reconocidos), así como las contingencias y restricciones del conocimiento que en ellas se produce. De este modo, se adjudica al conocimiento científico una "soberanía y autoridad sin límites".

A medida que este tipo de críticas a las instituciones y al conocimiento científico fueron ganando adherentes, otros autores, como Collins y Evans, plantearon una nueva preocupación. Según estos autores, el "problema de la legitimidad" estaba siendo reemplazado erróneamente por el "problema de la extensión". Al mismo tiempo que se cuestionaba la autoridad sin límites de la ciencia se planteaba la necesidad de ampliar el ámbito de la toma de decisiones políticas que involucraban de algún modo cuestiones científicas, para incluir nuevos actores más allá de la elite técnicamente calificada, con el propósito de fortalecer la legitimidad política de las decisiones. Esto habría derivado en "una tendencia a disolver los límites entre los expertos y el público de manera que ya no existe una base para limitar la extensión indefinida de los derechos de toma de decisiones técnicas" (Collins \& Evans 2002, p. 235). La preocupación de los autores es que, al socavar las fronteras entre ciencia y sociedad, no se pueda establecer diferencia alguna entre las creencias y los argumentos científicos, es decir que esta tendencia podría derivar en un relativismo extremo.

Según Harding, la discusión parece siempre polarizarse y terminar en la misma pregunta: “objetivismo o relativismo: ¿de qué lado estás?” (Harding 1995). Tanto para Harding como para Haraway es necesario superar esta polarización y, para ello, proponen repensar la noción misma de objetividad. Según estas autoras, para criticar el autoritarismo de las visiones tecno-científicas hegemónicas, no hace falta acudir al relativismo. Para Haraway, el relativismo es un espejo de la totalización en las doctrinas hegemónicas del objetivismo, dado que es un modo de no estar en ningún

\footnotetext{
2 Aspectos de un problema sobre los cuales nadie es consciente y/o que simplemente no han sido investigados.

3 No siempre es posible predecir cómo va a funcionar un sistema de producción de riesgo porque su funcionamiento depende en gran parte del comportamiento social (no controlado).
} 
sitio mientras se pretende igualmente estar en todas partes. "La igualdad de posicionamiento es una negación de responsabilidad y de búsqueda crítica" (Haraway 1991, p. 329).

Harding, por su parte, propone reemplazar esta pregunta por otra: “¿es posible repensar y actualizar la noción de objetividad y volverla útil para proyectos de producción de conocimiento contemporáneos?". Como respuesta a esta pregunta desarrolla una nueva doctrina "útil" pero no "inocente" de la objetividad, plasmada en lo que ella llama la objetividad fuerte. La principal diferencia con las nociones tradicionales es que esta doctrina busca desligar la noción de "objetividad" de los ideales de "neutralidad" y "universalidad". En este mismo sentido, Haraway afirma: "lucho a favor de políticas y de epistemologías de la localización, del posicionamiento y de la situación, en las que la parcialidad y no la universalidad es la condición para que sean oídas las pretensiones de lograr un conocimiento racional" (Haraway 1991, p. 335). Desde esta concepción, se insiste en la parcialidad de todas las formas de visión contra el mito de una visión universal: el conocimiento es siempre situado y la única posibilidad de encontrar una visión más amplia es ubicándonos en algún sitio en particular. Según Haraway (1991) la alternativa al "autoritarismo" científico y al relativismo extremo son los conocimientos parciales, localizables y críticos, que admiten la posibilidad de conexiones -solidaridad en la política o conversaciones compartidas en la epistemología-.

En esta intención de construir un conocimiento situado, los puntos de vista de los subyugados ocupan un lugar preferencial porque se asume que tienen más posibilidades de cuestionar, desafiar, y poner en duda el conocimiento establecido". Según Harding (1995), en las sociedades organizadas jerárquicamente como las nuestras, las actividades cotidianas de las personas que se encuentran en los grupos "privilegiados" o "dominantes" tienden a establecer ciertos límites a sus pensamientos, límites que no son creados por las actividades cotidianas de las personas que se encuentran en los grupos "subyugados". Las actividades administrativo-gerenciales son la forma de "gobernar" en las sociedades modernas contemporáneas; y los marcos conceptuales de las personas de los grupos dominantes son moldeados o influidos por estas prioridades administrativo-gerenciales. Estas prioridades permiten obtener la información que los administradores necesitan para funcionar efectivamente, pero también distorsionan y limitan nuestra comprensión crítica de las interacciones sociales, los valores e intereses que sostienen y constituyen los proyectos conceptuales dominantes.

En este sentido, para lograr una visión crítica de las interacciones, valores e intereses de los proyectos conceptuales dominantes, los pensamientos o proyectos de investigación deben partir desde "afuera" de estos esquemas conceptuales y las actividades que los generan: desde la vida de los excluidos - "las vidas marginales"-. Esto no significa que se busque exclusivamente comprender mejor las vidas marginales (por ejemplo, de las mujeres). La idea es generar proyectos de investigación que partan desde estas posiciones (sin que ello involucre que tomen como "verdad" lo que la gente en esas posiciones piensa o dice) para explicar no solo esas vidas, sino también el orden social y natural mismo, tanto a nivel micro como macro (lo cual incluye las relaciones del hombre con la naturaleza y las filosofías que han sido desarrolladas para explicar las ciencias). Según Harding (1995), los enfoques posicionales proveen un mapa, un método, para maximizar la objetividad fuerte que es más efectiva para proyectos de investigación que se enfrentan con ciencias que han sido construidas y moldeadas por los valores e intereses de los grupos sociales dominantes. En palabras de Haraway, los conocimientos situados "prometen versiones transformadoras más adecuadas, sustentadas y objetivas del mundo" (Haraway 1991, p. 328).

Retomando el dilema de Moore et al. (2011), creemos que los enfoques posicionales propuestos por Harding y Haraway podrían inspirar una tercera vía "superadora" de las dos opciones supuestamente dicotómicas propuestas. Y es justamente ésta la opción que planteamos desde el grupo "Epidemiología de la Ciencia Digna", que exponemos en la sección cuatro. 


\section{Reflexiones sobre el estado actual de los saberes académicos y las resistencias sociales relativas a la expansión del modelo de la agrobiotecnología}

Si bien las relaciones entre ciencia y tecnología han sido concebidas e interpretadas de diversas maneras por distintos autores (véase Niiniluoto 1997), en las últimas décadas se ha generalizado la noción de que tal distinción resulta difícil de delimitar (Rodríguez Alcázar 2009).

Así, por ejemplo, el concepto de tecno-ciencia, ha ganado consenso como descriptor de la dinámica hegemónica en esta etapa, caracterizada por el desarrollo de nuevos cuerpos de conocimientos integrados, en que los aspectos científicos y los técnicos se encuentran profundamente articulados en todas las etapas del proceso de construcción (Hottois 1999). Este vínculo íntimo se pone de manifiesto en aspectos tales como la declaración de ambiciosas "promesas" tecnológicas para la obtención de importantes inversiones destinadas a investigaciones. En el mismo sentido, la justificación de estas inversiones se refuerza a través de la adjudicación de valores pretendidamente positivos al contexto tecnológico, y de este modo la tecno-ciencia no solo promueve nuevas tecnologías, sino que también legitima el propio sistema productivo al que sirve de base.

Sin duda, una de las áreas tecno-científicas que han presentado un mayor impacto sobre las sociedades contemporáneas ha sido la de la biotecnología aplicada a la producción de paquetes tecnológicos basados en cultivos genéticamente modificados (GM), problemática en la que se centra este trabajo.

\subsection{Tecnociencia, extractivismo y agricultura transgénica en Latinoamérica: su legitimación en el discurso hegemónico}

El papel de la tecno-ciencia en la actual crisis socio-ambiental resulta un aspecto clave para contextualizar la problemática abordada. En términos de la declaración de la recientemente constituida Unión de Científicos Comprometidos con la Sociedad y la Naturaleza en América Latina (UCCSNAL):

El conocimiento científico y tecnológico, en particular aquel desarrollado en un contexto reduccionista, sin el debido control social, ha contribuido a crear problemas ambientales y de salud, con alcances muchas veces catastróficos e irreversibles (UCCSNAL 2015)

En particular, en América Latina, la expansión del extractivismo y el agronegocio han abonado la actual crisis social, ambiental y sanitaria, sometiendo a nuestros territorios y a sus habitantes a un incesante despojo y extinción (Svampa 2011, 2012a, 2012b). Al mismo tiempo, desde el discurso dominante, se asignan a las soluciones científico-tecnológicas un rol cada vez más preponderante en la resolución de las crisis, ocultando la discusión ético-política de fondo, situando a los científicos, expertos y tecnócratas en el rol de autoridades y soslayando la indispensable participación de toda la sociedad en la toma de decisiones en problemáticas que involucran a la ciencia y la tecnología (Wynne 2001).

Todo ello en un escenario en que, la formación de científicos se caracteriza cada vez más por la fragmentación y la especialización de saberes. En relación con ello Levy Leblond (2003) plantea que, como resultado de dicha fragmentación, la ciencia contemporánea se ha desvinculado de la cultura, se ha enajenado y ha pasado a construir conocimientos efímeros, caracterizados por una pérdida creciente de pertinencia y relevancia.

En particular, en el ámbito del conocimiento biológico, la hegemonía del llamado "discurso de acción de los genes" (Fox Keller 1995) ha contribuido a naturalizar una conceptualización de la biología en extremo reduccionista e ingenua, que opera como legitimador epistemológico de posturas que promueven acríticamente los desarrollos tecno-científicos de la biotecnología, al mismo tiempo que ocultan sus riesgos. Asimismo, como analizaremos más adelante, los modelos epidemiológicos 
clásicos enmascaran los impactos en la salud de los problemas ambientales y legitiman la ausencia de daños.

En nuestra región, la debilidad de una corriente crítica que no fue capaz de instalar el debate político y epistemológico en torno a estos temas y la escasa participación social, signaron un proceso de rápida expansión del modelo. Todo ello, sumado a las agresivas políticas de comercialización de las empresas de agrobiotecnología, a la complicidad de los funcionarios y a la debilidad o la inexistencia de marcos regulatorios. En ausencia de debate social, el modelo se estableció y creció acompañado de un discurso exitista que lo presentó como virtuoso. Este discurso, que reproduce la lógica que las corporaciones decidieron instalar a nivel global, asume que la tecnología es la "estrategia de salvación de la humanidad", presenta al modelo de producción con OGM como inherentemente progresivo y refuerza la noción de que esta tecnología representa un bien en sí mismo. Así, la expansión de los OGM se explica no sólo como una oportunidad beneficiosa, sino que, incluso, se la presenta como una estrategia única y necesaria para cubrir los requerimientos nutricionales de la población mundial, naturalizando la idea del "inexorable curso del avance tecnológico" (Folguera et al. 2013).

Estos argumentos no sólo forman parte de las estrategias publicitarias de las corporaciones, sino que se expresan en diversos documentos de organismos internacionales, como la Organización para la Cooperación y el Desarrollo Económico (OCDE 2009) y la Food and Agriculture Organization (FAO 2000, 2009), que promueven lo que denominan como una nueva "bioeconomía". Este concepto, que está cada vez más presente en diversos discursos intentando mimetizarse con modos "amigables" de vinculación entre producción y naturaleza; involucra la promoción de diversas tecnologías que apuntan a la privatización y mercantilización de las especies vivientes, los ecosistemas y los procesos que constituyen y sostienen la trama de la vida:

La bioeconomía es un proyecto y al mismo tiempo una visión situada dentro de la narrativa neoliberal del crecimiento económico y de la competitividad y habla de una nueva economía basada en la manipulación, explotación y apropiación tecnológica de la materia viviente (Pavone 2012, p. 1).

Estos y otros organismos internacionales celebraron y apoyaron la implementación de las innovaciones biotecnológicas en agricultura basadas en la biotecnología, argumentando que esto ayudaría a resolver los principales problemas de producción de alimentos y salud que enfrentará la humanidad en el futuro próximo (Arancibia 2012):

Los recientes avances en las aplicaciones agrícolas de la biotecnología moderna muestran un potencial significativo para contribuir a una mejora sostenible de la productividad agrícola (OECD 2009, p.15). De aquí a 2030, la población mundial habrá crecido hasta los 8,3 billones [...] pero al mismo tiempo la gran mayoría de los ecosistemas mundiales están ya sobre-explotados y de una forma insostenible [...] la biotecnología puede ofrecer la solución para muchos de los problemas de salud y alimentos que afectan al plantea (OECD 2009, p. 8).

Según la FAO (2000) y la Organización Mundial de la Salud (2012) los transgénicos son una buena solución frente al problema del hambre en el mundo, especialmente para alimentar a poblaciones vulnerables:

La FAO reconoce que la ingeniería genética puede contribuir a elevar la producción y productividad en la agricultura, silvicultura y pesca. Puede dar lugar a mayores rendimientos en tierras marginales de países donde actualmente no se pueden cultivar alimentos suficientes para alimentar a sus poblaciones (FAO 2000).

Somos conscientes de que aumentar la productividad agrícola es el principal medio para satisfacer la demanda creciente de alimentos (FAO 2009, p. 5).

El optimismo expresado en estas posturas se acompaña de la negación de los riesgos involucrados. Por ejemplo, en referencia a los alimentos genéticamente modificados actualmente disponibles en el mercado internacional, la Organización Mundial de la Salud ha afirmado que "no es probable que presenten riesgos para la salud humana" (Organización Mundial de la Salud 2012). Esta afirmación se 
realiza aun sin contar con estudios epidemiológicos de mediano y de largo plazo que así lo demuestren, en una muestra de confianza que nada tiene que ver con la lógica de la ciencia.

En Argentina, en términos generales, los científicos y voceros de instituciones de ciencia y técnica, encarnan los ejes de este discurso. A su vez, se resguardan de posibles críticas y responsabilidades reproduciendo la imagen del tecno-científico como mero descriptor del mundo, asumiendo que su tarea y sus producciones son neutrales, de modo que las decisiones políticas, las consecuencias sociales y los aspectos éticos quedan soslayados, o confinados exclusivamente al ámbito de las aplicaciones. En el caso de los impactos producidos por los agroquímicos, esta postura ideológica ha dado lugar al llamado discurso de las "buenas prácticas", que atribuye los daños producidos al "mal uso" de estos insumos, restringiendo la discusión al ámbito regulatorio y en última instancia culpabilizando a las víctimas por los daños producidos.

Otro pilar del discurso legitimador del modelo de producción basado en OGM asumido por científicos y funcionarios es la confianza en que no sólo no generará impactos negativos, sino que los efectos beneficiosos favorecerán a toda la sociedad, desestimando sistemáticamente los riesgos y daños potencial o efectivamente involucrados. La confianza acrítica como punto de partida resulta en que, aun en los casos en que se admita la necesidad de realizar más investigaciones para evaluar los riesgos, no se contemple la necesidad de detener las aplicaciones tecnológicas hasta tanto se demuestre la inocuidad, lo cual sería la conducta pertinente conforme a lo previsto por el "principio precautorio" (CONICET 2009). Por el contrario, el discurso hegemónico apunta a invertir la lógica del principio precautorio, esto es: se reclama que los estudios independientes o incluso las propias víctimas demuestren que esta tecnología causa efectivamente algún tipo de daño.

Todo ello en un escenario en que el marco regulatorio es fragmentado y permeable a la rápida introducción de estas innovaciones. Por ejemplo, el ente responsable de la regulación de agroquímicos en Argentina, SENASA, ${ }^{4}$ depende del Ministerio de Agricultura. Notablemente, el Ministerio de Salud y la Secretaría de Medio Ambiente no tienen injerencia en las políticas regulatorias. La resolución de aprobación de cada producto y las pruebas científicas que la justifican, no están accesibles al público. Según la legislación vigente, el organismo tiene la responsabilidad de aprobarlos, clasificarlos toxicológicamente y fijar normas de uso. Pero en la práctica sólo se ocupa de las dos primeras y transfiere la responsabilidad de la regulación del uso a las provincias. Aquí hay dos puntos clave: 1) las provincias que establecen restricciones a la aplicación de agroquímicos basan sus normativas en la clasificación toxicológica provista por SENASA, y 2) esta clasificación sólo toma en cuenta los posibles daños letales agudos (la muerte en un corto período de exposición). Ello implica que se soslayan todos los posibles daños crónicos y sub-letales en un largo período de exposición, tales como cáncer, malformaciones fetales y abortos espontáneos, entre otras patologías, los cuales son ya evidentes para millones de argentinos expuestos a los agroquímicos directamente por fumigaciones, o indirectamente por el agua de lluvia o los alimentos que se consumen tanto en zonas rurales como urbanas. En este sistema regulatorio, el glifosato (Roundup Ready), por ejemplo, es clasificado como producto "de baja toxicidad”, por lo cual casi no existen limitaciones provinciales a su aplicación. Así, se utilizan más de 250 millones de litros anuales de este herbicida sin restricciones, fumigándolo hasta la puerta de las casas, o rociándolo con aviones sobre poblados, escuelas rurales y fuentes de agua.

Ante la eventualidad de nueva información sobre riesgos de un producto, la normativa de SENASA prevé una revisión de su aprobación y clasificación toxicológica, lo que puede derivar en la prohibición o restricción de su uso. Sin embargo, la decisión de iniciar dicha revisión sólo está en manos del equipo técnico de SENASA. De este modo, en el caso del glifosato, tal revisión nunca se inició aun frente a resultados contundentes de investigaciones independientes que demuestran diversos tipos de daños no tomados en cuenta por la normativa vigente, ni tampoco frente el reciente informe de la Agencia Internacional para la Investigación del Cáncer (IARC) de la OMS (Marzo 2015), según el cual el glifosato "puede provocar cáncer en seres humanos".

\footnotetext{
${ }^{4}$ Servicio Nacional de Sanidad y Calidad Agroalimentaria.
} 
En cuanto al debate científico sobre OGM y agroquímicos en Argentina, es necesario destacar que ha sido y aun es sumamente limitado, y no ha encontrado su espacio en las instituciones de la ciencia y la tecnología. Menos aún en el ámbito filosófico. Mientras el discurso hegemónico de los especialistas en biotecnología es omnipresente en ámbitos educativos, científicos y en medios de comunicación, las posturas críticas son débiles, aisladas y sistemáticamente desestimadas y/o silenciadas.

\subsection{La acción de comunidades y científicos comprometidos en Argentina}

Mientras que el discurso científico hegemónico promueve el avance de la agrobiotecnología en Argentina, se multiplican las denuncias de poblaciones rurales sobre graves problemas de salud a partir del uso masivo de glifosato y otros agroquímicos utilizados en los cultivos GM. Estas denuncias fueron generalmente ignoradas por los sectores académicos promotores del modelo, así como por los medios de comunicación y los organismos gubernamentales encargados de velar por la salud pública. Expuestas a la lógica de la inversión del principio precautorio, las poblaciones afectadas se vieron obligadas a darle entidad pública a los problemas de salud que padecían como resultado de la degradación de su ambiente, produciendo evidencia del daño por sus propios medios y/o solicitando la asistencia de especialistas provenientes de ámbitos académicos.

Una de las experiencias más emblemáticas, que podría encuadrarse en la metodología conocida como "epidemiología popular" (Brown 1987, Brown \& Mikkelsen 1990), fue la realización de encuestas epidemiológicas por parte de los mismos vecinos. Un grupo de madres de un barrio limítrofe a las plantaciones de soja en las afueras de la Ciudad de Córdoba, que luego fueron conocidas como "Madres de Ituzaingó", iniciaron un relevamiento en 2001 cuando una vecina advirtió que muchas mujeres en el pueblo llevaban pañuelos para cubrir sus cabezas y muchos niños usaban barbijo. Buscaron así determinar si realmente eran "demasiados" los enfermos y pedir ayuda a las autoridades. Durante cuatro meses recogieron datos puerta a puerta sobre el estado de salud de los habitantes, armando un mapa de localización de enfermedades. Encontraron un número extraordinario de padecientes de enfermedades poco frecuentes que se vinculan, entre otros determinantes, con alteraciones ambientales (fundamentalmente diversos tipos cáncer poco frecuentes, enfermedades de la piel y enfermedades autoinmunes). Con estos datos, las madres presentaron la lista y el mapa de localización de los enfermos al Ministerio Provincial de Salud, pero no obtuvieron ninguna respuesta. Entonces comenzaron a organizar marchas en las calles del pueblo y recién después de que un canal de televisión local mostrara una de sus manifestaciones, el Ministerio Provincial de Salud las recibió y les prometió llevar a cabo un estudio interdisciplinario del medio ambiente y otro sobre incidencia de cáncer.

Si bien el estudio de medio ambiente reveló la presencia de pesticidas en agua y aire, el estudio de incidencia de cáncer indicaba que las cifras locales estaban dentro de los límites de las cifras esperadas estadísticamente. ${ }^{5}$ Sin embargo, conforme a la evaluación de las madres, el estudio oficial era deficiente porque no censó la totalidad de los casos. Ante esta situación las madres pidieron ayuda a la Fundación para la Defensa del Ambiente, coordinada por el Dr. en Biología Raúl Montenegro. El mismo denunció haber tenido acceso a un estudio de suelo de una agencia oficial -no publicado- que demostraba alto grado de contaminación con agroquímicos y presentó los resultados en una conferencia de prensa. Tras muchas disputas, las madres lograron que se emitieran tres Ordenanzas Municipales que declaraban el Estado de Emergencia Sanitaria del barrio y prohibían la fumigación aérea y terrestre de agroquímicos en los campos lindantes al barrio. Sin embargo, los productores no respetaban las restricciones. Se abrió entonces una etapa de producción de estudios por las diversas partes que probaban o que desestimaban la contaminación (6 estudios oficiales y 3 estudios independientes) (Arancibia 2013, 2015).

Las constantes violaciones a las ordenanzas llevaron a las madres a apelar al poder judicial. Presentaron demandas judiciales, apuntando a los dueños de los campos lindantes por violación a la normativa y por responsabilidad sobre la contaminación y enfermedades de los vecinos. La resolución

${ }^{5}$ Las cifras oficiales daban cuenta de menos casos que los relevados por las madres. 
judicial fue positiva para las madres, pero se hizo esperar: recién en 2009 la corte provincial dio lugar a un recurso de amparo que prohibía expresamente que los dueños de los campos lindantes siguieran usando agroquímicos. Y en 2012, tras un juicio histórico, uno de estos mismos productores y un dueño de un avión fumigador fueron condenados a prisión condicional, por violar las ordenanzas restrictivas y el mencionado recurso de amparo, poniendo en riesgo la salud pública. En su camino de resistencia, en un comunicado que divulgaron las Madres planteaban:

Nuestro barrio de baja condición social debe soportar múltiples factores de degradación y de contaminación ambiental que afecta de forma directa el pleno goce y el ejercicio de los derechos humanos. Es un ícono de la problemática ambiental de la mayoría de las ciudades y asentamientos humanos que se encuentran en las zonas aledañas a cultivos de soja [...]. Este país, laboratorio forrajero, encontró sus mejores defensas en mecanismos sutiles de ocultamiento y de invisibilidad, de allí que nuestro mayor esfuerzo sea siempre el de hacer visible lo invisible, y en medio de la fiesta obscena de las exportaciones récord y de la evasión de retenciones, exhibir sus consecuencias trágicas en el hambre y en las pérdidas de vida que el modelo provoca (Grupo de Madres de Ituzaingó de Córdoba 2005, p. 9).

En 2004, las madres viajaron a Buenos Aires para interpelar a las autoridades nacionales. Allí se conectaron con el Grupo de Reflexión Rural, y junto con ellos, Centro de Protección a la Naturaleza (CEPRONAT) y Unión de Asambleas Ciudadanas organizaron la primera campaña nacional "Paren de Fumigar". El objetivo era promover la organización de los vecinos rurales, frenar las fumigaciones y promover un cambio de sistema agro-productivo. Así, se crearon múltiples asambleas vecinales, las cuales junto con médicos rurales y algunos científicos solidarizados recolectaron evidencia de las enfermedades. En 2009 estas experiencias se publicaron en el libro "Pueblos fumigados" (Rulli 2009). En 2010 se realizó el $1^{\circ}$ Congreso Nacional de Médicos de Pueblos Fumigados, en la Universidad Nacional de Córdoba, donde presentaron varios estudios sobre los efectos de los agroquímicos. La difusión de estos estudios sirvió para instalar el problema de las fumigaciones en el ámbito público, promover legislaciones restrictivas del uso de algunos agroquímicos en el ámbito municipal, así como resolver casos judiciales individuales a favor de vecinos denunciantes en diversas localidades del país. En el año 2011 se realizó en la Facultad de Ciencias Médicas de Rosario, el 1er Congreso Latinoamericano de Salud Socioambiental, en el cual la presencia de actores vinculados a los pueblos fumigados, junto a científicos y referentes de las ciencias médicas y del derecho, hicieron evidente los vínculos entre los modelos extractivistas y los problemas de salud de las comunidades lindantes con ellos. Este espacio se transformó en un ámbito de encuentro e intercambio entre diversos actores, con una frecuencia bianual bajo la continencia de un ámbito académico Universitario (Verzeñassi 2016). De este modo, se multiplicaron diversos tipos de estudios independientes (experimentales, epidemiológicos, clínicos, eco-toxicológicos) sobre efectos de pesticidas en la salud y el ambiente. También se repitieron experiencias de "epidemiología comunitaria" en otras localidades del país y, como veremos en el siguiente apartado, desde la Universidad Nacional de Rosario y la Universidad Nacional de Córdoba se desarrollaron nuevas iniciativas para producir datos epidemiológicos junto con las comunidades.

Pese a ello, las investigaciones independientes siguen siendo escasas y en la mayoría de los casos los investigadores no cuentan con el apoyo de sus instituciones para desarrollarlas, difundirlas y/o profundizarlas. Más allá de algunos avances en las legislaciones a nivel local, a nivel nacional no hay reconocimiento oficial del daño a la salud que generan los principales agroquímicos utilizados en Argentina, no ha habido cambios en las regulaciones nacionales, ni se aplica el principio precautorio. A su vez, la comunicación pública de los resultados de las investigaciones independientes generó grandes controversias y fuertes resistencias. El ejemplo más representativo es el de las investigaciones del Dr. Andrés Carrasco sobre los efectos teratogénicos del glifosato, que desataron una violenta reacción de las empresas, los funcionarios públicos de las instituciones de CyT, y de diversos miembros de la comunidad científica cooptada por las corporaciones, dando lugar a una intensa campaña de desprestigio y hostigamiento que apuntaba a su silenciamiento. 


\section{La propuesta del Grupo de Epidemiología de la Ciencia Digna}

¿Cómo va a responder nuestra comunidad científica ante el clamor por la defensa de la vida de los seres humanos de las ciudades y del campo? ¿Cómo evitar que la ciencia termine sirviendo al juego de la Hegemonía? Es decir, denunciando sin revelar, informando sin movilizar, enfocando factores aislados la problemática, sin mostrar su relación con los procesos estructurales que los generan (Breilh 2010, p. 84).

Recuperando diversas experiencias de producción de un conocimiento que va a contrapelo de las instituciones dominantes y buscando alternativas superadoras, en 2015 se conformó el Grupo de Epidemiología de la Ciencia Digna ${ }^{6}$ en el que confluyeron tres grupos previamente conformados, con trayectorias diversas.

Uno de ellos es el equipo de la Práctica Final de la carrera de Medicina y el Instituto de Salud Socio Ambiental, de la Facultad de Ciencias Médicas de la Universidad Nacional de Rosario, en Santa Fe. A través de la realización de Campamentos Sanitarios el equipo investiga qué sucede con la salud de aquellas comunidades rurales de menos de 10.000 habitantes (adonde no llegan los registros del Estado) de la región. En los Campamentos Sanitarios durante cinco días los estudiantes de último año de la carrera relevan el estado de salud de poblaciones rurales expuestas a distintos factores de contaminación. Acompañados por sus tutores, los estudiantes visitan casa por casa para realizar una encuesta sobre la salud de todos los integrantes de cada hogar. Al mismo tiempo, hacen un examen clínico de la salud de los niños escolarizados, y realizan diversos talleres de promoción de la salud en plazas y escuelas. El último día del campamento, se reúnen con los habitantes en un espacio público para compartir los resultados principales obtenidos. Desde 2010 se han realizado Campamentos Sanitarios en 23 localidades de la Provincia de Santa Fe y 4 localidades de otras provincias. De este modo, se han relevado datos de salud de más de 96.800 personas. A partir de esta información, los vecinos han podido sustentar diversos reclamos relacionados con su salud socio-ambiental, al tiempo que las autoridades y profesionales de la salud recomenzaron a contar con información que podría servir de base para re-direccionar la política de salud a nivel local. Los resultados de varios de estos Campamentos han sido presentados en los Congresos Internacionales de Salud Socioambiental, así como en congresos de la Asociación Latinoamericana de Medicina Social (ALAMES) entre otros espacios académicos, así como en ámbitos de convocatoria de movimientos sociales y en el Parlamento Argentino.

Otro grupo surgió en el marco de un proyecto de Observatorio Ambiental y Epidemiológico de Poblaciones Expuestas a Agroquímicos de la Universidad Nacional de Córdoba, actualmente con un perfil independiente, que apunta a realizar relevamientos epidemiológicos similares a los de Santa Fe con un enfoque de epidemiología comunitaria. Hasta ahora este equipo ha realizado relevamientos en las localidades de Morrison y Pozo del Molle, en la provincia de Córdoba, con una novedosa modalidad de trabajo que involucra la formación de equipos interdisciplinarios en los que participan investigadores y profesionales de diversas disciplinas (medicina, sociología, estadística, biología, psicología social, biofísica) junto a vecinos (Arnuplhi et al. 2014). La iniciativa del relevamiento surgió en ambas localidades de un grupo de vecinos auto-organizados para defender la salud de su comunidad, quienes acudieron a asambleas del movimiento Paren de Fumigar para pedir ayuda. Allí se encontraron con algunos de los investigadores de la universidad y decidieron comenzar a trabajar juntos. En este caso los vecinos también realizaron encuestas, junto con los profesionales. Por otro lado, una vez terminados los relevamientos el equipo de profesionales continuó su trabajo con la comunidad realizando talleres de promoción de salud, de agroecología y foros ciudadanos para negociar el diseño e implementación de nuevas regulaciones que protejan la salud de los vecinos.

El tercer grupo se constituyó en Buenos Aires, integrado por miembros de diversas disciplinas Biológicas, Sociales y Médicas con el propósito inicial de brindar apoyo técnico a los dos primeros grupos presentados y contribuir a su articulación.

\footnotetext{
${ }^{6}$ Los autores de este trabajo somos integrantes del Grupo de Epidemiología de la Ciencia Digna.
} 
A partir de la convergencia de estos tres grupos, en 2015 se constituyó el Grupo de Epidemiología de la Ciencia Digna, cuyo objetivo es contribuir a hacer visibles los problemas de salud que presentan poblaciones expuestas a riesgos y daños socio-ambientales y promover la auto-organización popular para hacer frente a estos problemas, en busca de generar un cambio. El camino planteado para articular y fortalecer las experiencias en curso involucra la construcción de nuevas herramientas para que las comunidades puedan conocer y evaluar su estado de salud. La construcción de estas herramientas se plantea en el marco de una perspectiva de "epidemiología comunitaria", con el propósito de coproducir nuevos datos epidemiológicos entre científicos y comunidades, así como nuevas y diversas propuestas de acción. Esta estrategia buscó desde sus orígenes no quedar encerrada en la lógica de los modelos y metodologías reduccionistas de la ciencia hegemónica, ya que la problemática planteada es compleja y no reconoce la lógica lineal de una causalidad única y no puede limitarse a un análisis exclusivamente cuantitativo. Por ello, un aspecto relevante en este proceso de construcción es el análisis crítico de las metodologías y modelos clásicos, especialmente aquellos empleados en epidemiología.

A pesar de que en las últimas décadas en el campo de la epidemiología han surgido diversas corrientes críticas, en nuestro país, tanto el ámbito de los estudios gubernamentales como el de la investigación académica, están ampliamente dominados por la epidemiología empírica analítica. La posibilidad de incorporar los debates más recientes se encuentra obturada debido a que, usualmente, los promotores de estos enfoques niegan la necesidad de una discusión acerca de las bases conceptuales de las cuales parten, asumiendo a la evaluación "clínico experimental" como la única posibilidad legítima para este tipo de análisis. Así, las bases y categorías utilizadas no son objeto de revisión y expresan una fuerte inercia teórica. Ello se manifiesta, por ejemplo, en que las variables más frecuentemente utilizadas -son las de "riesgo" "carga" y "daño"-, cuya definición se remonta a los orígenes de la medicina laboral. Desde el punto de vista metodológico, en el enfoque asumido en esos trabajos podemos identificar una serie de operaciones reductivas cuyas consecuencias en los análisis finales será variable (pero innegable) de acuerdo con el caso analizado. Uno de los criterios fundantes es aquel que asume la homologación lineal de magnitudes cuantitativas de determinados indicadores (usualmente moleculares) como único legitimador del registro de un individuo enfermo. Conforme a este criterio, sólo la presencia de dicho indicador, manifestándose con una magnitud determinada, avalaría el registro del padecimiento de la enfermedad a la cual identifica. Estos supuestos involucran un triple reduccionismo, de modo que el análisis sólo reconocerá como enfermos a un limitado grupo que comprende: A. a los pacientes diagnosticados; B. a los pacientes cuyo diagnóstico se sustente en un indicador bioquímico; y C. sólo a aquellos en los que ese indicador se encuentre en un determinado rango cuantitativo. La presencia de este triple sesgo suele soslayarse a la hora de interpretar resultados y sacar conclusiones en los estudios epidemiológicos clásicos.

A su vez, es importante destacar que en la actualidad, la mayoría de los estudios epidemiológicos institucionales en nuestra región se realizan a partir de registros obtenidos en establecimientos de salud tales como hospitales o centros de salud periféricos. En relación con ello cabe señalar que este sistema de registro no tiene en cuenta qué ocurre con los ciudadanos que no pueden acceder al sistema de atención. A su vez, en el caso de aquellos que para hacerlo deben trasladarse a otras localidades, frecuentemente, la falta de registro de los lugares de origen impide relacionar los datos de salud con su localización territorial y, por tanto, con los factores ambientales que podrían estar interviniendo en los procesos de salud y enfermedad. Por ejemplo, las tasas de incidencia de cáncer o de anomalías congénitas que surgen de estudios epidemiológicos oficiales en la región del centro de Argentina donde se emplean grandes cantidades de agroquímicos, no son superiores a las esperadas estadísticamente en términos de los estándares internacionales, lo cual indicaría que no se estaría frente a daños ambientales específicos. ${ }^{7}$ Sin embargo, estos datos provienen de la casuística que se registra en los hospitales y maternidades de las grandes ciudades a las que, en muchos casos, no llegan

\footnotetext{
7 Datos del RENAC Registro Nacional de Anomalías Congénitas y del INC (Instituto Nacional del Cáncer), ambos organismos pertenecientes al Ministerios de Salud de la Nación
} 
los vecinos de los pequeños pueblos fumigados, o de quienes -en caso de ser atendidos allí- no se tiene en cuenta sus lugares de origen, diluyéndose sus datos en el universo de todos los allí atendidos, que en su mayoría viven en las grandes ciudades.

Así, los estudios epidemiológicos convencionales, no situados en el territorio y sesgados por diversas decisiones reductivas, tienden a subestimar, invisibilizar o llanamente ocultar los daños en la salud que están experimentando las poblaciones afectadas por el presente modelo de producción con transgénicos. Daños que las propias poblaciones afectadas padecen y reconocen como tales desde hace años y que requieren ser reconocidos por el estado y por el conjunto de la sociedad. Para avanzar en dicha dirección consideramos que debemos recurrir a una lógica no reduccionista, que integre el conocimiento científico con los saberes y las experiencias que han adquirido estas poblaciones.

En nuestro país, el debate sobre las limitaciones, sesgos y consecuencias sociales y políticas de la concepción hegemónica en epidemiología no se ha desarrollado en medios académicos, incluso sectores caracterizados como "contra-hegemónicos" suelen reproducir esta perspectiva en sus marcos teóricos. En relación con la frecuente aplicación acrítica de criterios e indicadores cuantitativos para evaluar un fenómeno complejo, coincidimos con Bachelard en que "es preciso reflexionar para medir, antes que medir para reflexionar" (Bachelard 1979). Y en este sentido se impone la necesidad de asumir el desafío de la complejidad.

La complejidad se impone de entrada como imposibilidad de simplificar; ella surge allí donde la unidad compleja produce sus emergencias, allí donde se pierden las distinciones y claridades en las identidades y causalidades, allí donde los desórdenes y las incertidumbres perturban los fenómenos, allí donde el sujeto-observador sorprende su propio rostro en el objeto de observación, allí donde las antinomias hacen divagar el curso del razonamiento (Morin 1977, p. 377).

En función de ello, nos planteamos la necesidad de construir un conocimiento colectivo y situado, que asuma el desafío de la complejidad, que aporte a la toma de conciencia, a la visibilización del problema en el conjunto de la sociedad, que contribuya a la articulación entre diversas comunidades que están en situación similar y a su empoderamiento para trasformar esa realidad. A partir de estas y otras consideraciones decidimos desarrollar una herramienta que permita obtener información significativa sobre el estado de salud de las personas en su territorio, antes que en el centro de salud.

Asumiendo estos criterios y prioridades, nos centramos en el diseño de una encuesta de producción/recolección y análisis de datos sobre salud-socioambiental, que pueda ser aplicada y autogestionada en toda comunidad que desee hacerlo, con la intención de que el diseño original sea un puntapié inicial para generar una nueva versión más acabada en un proceso de interacción con las comunidades afectadas. En esta primera instancia, trabajamos conjuntamente investigadores y profesionales de distintos lugares del pais ${ }^{8}$ en la elaboración de una primera versión de esta encuesta epidemiológica. Ésta consiste en un cuestionario "básico", que apunta a explorar el siguiente interrogante: ¿de qué se enferma y de qué se muere la población en esta localidad? Este cuestionario irá acompañado de un instructivo de aplicación para que cualquier comunidad pueda utilizarlo de manera autogestiva. A su vez se prevé que a este cuestionario se podrán ir sumando sub-módulos específicos con nuevas preguntas para ser aplicados en diversos contextos, que permitan identificar asociaciones entre las patologías/muertes relevadas y diversos problemas medio-ambientales (ya sean producto de actividades productivas, de la incorporación de nuevas tecnologías, o de catástrofes ambientales). El formato de la herramienta propuesta es sumamente flexible: si bien los datos recabados mediante el cuestionario básico podrán dar lugar a análisis comparativos o integradores de diversas localidades, los módulos adicionales permiten adaptarla a las diversas necesidades sociales y ambientales específicas de las comunidades que deseen aplicarla.

Asumimos que si bien, de acuerdo con la lógica de la epidemiología clásica, esta metodología involucra una pérdida de especificidad en los diagnósticos, permite ganar sensibilidad en el análisis de las categorías a las que deseamos brindar mayor relevancia; priorizando la percepción de la población

\footnotetext{
${ }^{8}$ Tanto filósofos, sociólogos y antropólogos como profesionales de la salud y docentes en Medicina, biólogos de diversas áreas, físicos y geólogos.
} 
sobre su propia salud, en su territorio. En ese sentido, consideramos que si el relevamiento se limitara a un análisis cuantitativo y a una estadística descriptiva de datos epidemiológicos de la población involucrada, estaría faltando a la necesidad de que este proceso y sus resultados contribuyan efectivamente al empoderamiento de las comunidades. Por ello, si bien la propuesta mantiene aspectos del análisis cuantitativo-estadístico, incluye también instrumentos de producción de datos cualitativos igualmente jerarquizados -recabados a través de entrevistas semi-estructuradas-, cuyas guías de pautas se encuentran en proceso de desarrollo. Se prevé asimismo que tanto la encuesta cuantitativa como las entrevistas que recuperan la información cualitativa constituyan instancias entre otras a desarrollar, articulando estas herramientas como parte de un proceso que comprende espacios de trabajo colectivo previos y posteriores a su aplicación (tales como talleres, debates, mapeos ambientales). La integración de estas diversas modalidades de trabajo permitirá generar nuevos datos cualitativos, así como interpretar colectivamente toda la información producida en el proceso y contribuir a desarrollar estrategias de acción específicas y concretas en cada localidad.

Así, reconocemos un doble carácter de este proyecto, que supone la posibilidad de abordar diferentes escalas: por un lado, permite reunir datos epidemiológicos que podrían ser analizados y/o comparados a un nivel macro (regional, provincial, o nacional); y por otro, en el nivel local, la encuesta será aplicada en todas las comunidades que así lo decidan. En la primera escala, si bien el análisis de los datos no escapará completamente a las lógicas usuales de la epidemiología empírica analítica, las preguntas y registros se construirán en base a criterios distintos a los empleados en los estudios de centros de salud (ya que recogen las necesidades de las comunidades). En cuanto a la escala local, al aplicar la encuesta en comunidades que la demanden, los procesos transitados y el uso de los resultados se adaptarán a las características de cada comunidad particular. En relación con este segundo aspecto, queremos destacar que jerarquizamos la historicidad y la localización en los territorios de este proyecto: consideramos que cada comunidad que la aplique se habrá encontrado con la necesidad de una herramienta de estas características por caminos distintos y la empelará conforme a sus propósitos y modalidades. Así, la encuesta es parte de un proceso más amplio y diverso que la resignifica en cada caso.

Por otro lado, los datos de cada comunidad surgen de la aplicación de la encuesta por cuenta de los propios vecinos, lo cual necesariamente los posiciona en un rol activo y protagónico. En este sentido, destacamos que los datos obtenidos pertenecen a la comunidad, de modo que sólo serán integrados a un análisis de mayor escala si se cuenta con la debida autorización de los involucrados. En el caso de que los vecinos no deseen que sus datos se integren a la base de datos general, se respetará esta decisión, atendiendo a que de todos modos, si la comunidad así lo requiere, se brindará un análisis sistematizado de sus resultados para facilitar su interpretación.

Para finalizar, nos parece importante enfatizar que consideramos que la aplicación de la encuesta es una de las instancias de concientización y empoderamiento que podrían contribuir a fortalecer los procesos de auto-organización y lucha que las diversas comunidades desarrollan en sus territorios. En ese contexto, los protagonistas locales no sólo le conferirán sus propios sentidos, sino que también, en sus propios contextos de aplicación, serán ellos quienes decidan las modalidades y propósitos de la interpretación y comunicación posterior de esos datos, así como de las diversas actividades y estrategias que se presenten en el curso del proceso de resistencia que decidan transitar.

\section{Conclusiones}

- El discurso dominante asigna a las soluciones científico-tecnológicas un rol cada vez más preponderante en la toma de decisiones y resolución de las crisis relativas a la incorporación de nuevas tecnologías, ocultando la discusión ético-política, situando a los científicos y tecnócratas en el rol de autoridades neutrales y soslayando la indispensable participación de toda la sociedad en la toma de decisiones. 
- El debate científico y ético sobre OGM y agroquímicos en Argentina, ha sido y aun es sumamente limitado, y no ha encontrado su espacio en las instituciones de la ciencia y la tecnología ni tampoco en el ámbito público.

- El predominio del geneticismo en el ámbito del conocimiento biológico ha contribuido a naturalizar una conceptualización reduccionista, que legitima los desarrollos tecnocientíficos de la biotecnología, al mismo tiempo que oculta sus riesgos; siendo esta postura mayoritaria entre las voces académicas que se expresan en torno al modelo de la agrobiotecnología.

- Los modelos epidemiológicos clásicos enmascaran los impactos en la salud de los problemas ambientales y legitiman la ausencia de daños.

- Los grupos de investigación que abordan la problemática de riesgos, impactos y daños del modelo en la salud y el ambiente son débiles, aislados y sistemáticamente desestimados, hostigados y/o silenciados.

- Los científicos y voceros de instituciones de ciencia y técnica, presentan al tecnocientífico como mero descriptor neutral del mundo, confinando los aspectos éticos, culturales y políticos al ámbito de las aplicaciones, dando lugar al llamado discurso de las "buenas prácticas" que restringe la discusión sobre la adopción de nuevas tecnologías al ámbito regulatorio y culpabiliza a las víctimas por los daños producidos.

- A su vez este discurso hegemónico apunta a invertir la lógica del principio precautorio, esto es: se reclama que los estudios independientes o incluso las víctimas demuestren que esta tecnología causa efectivamente algún tipo de daño.

- Frente a estas tensiones, son las comunidades afectadas las que intentan hacer visibles los daños que ocasiona el modelo, en sus propios territorios, demandando en ocasiones apoyo de las instituciones académicas. Compartimos con Harding y Haraway la noción de que los puntos de vista de los subyugados son los que tienen mayor capacidad de visión crítica sobre los modelos y esquemas conceptuales dominantes.

- Es necesario tender puentes entre las comunidades en resistencia y los ámbitos académicos, pero a la vez es indispensable interpelarnos acerca de qué tipo de conocimiento es aquel que puede contribuir al empoderamiento de las comunidades.

- En ese camino, señalamos la necesidad de generar herramientas político-cognitivas y estrategias de nuevo tipo, coproducidas entre los científicos críticos y las comunidades afectadas, que superen la lógica reduccionista y fragmentaria de la ciencia hegemónica, que produzcan un "conocimiento situado" e integren saberes de diferente naturaleza y que dialoguen con diferentes lógicas: la interdisciplinaria, los saberes no científicos, la dimensión política, ética, cultural, ideológica y ambiental, motorizadas por valores tales como la solidaridad, la equidad, la protección de los bienes comunes de la naturaleza, la salud comunitaria de las actuales y las futuras generaciones.

\section{Preguntas y desafíos abiertos por el proyecto}

Quedan por abordar aún aristas no resueltas que transcurren entre los marcos teóricos ideales y la tendencia a asumir una postura pragmática en contextos de necesidades urgentes.

- ¿Cómo romper la idea perversa de la inversión del principio precautorio? Debe quedar claro que no se trata de que las víctimas tengan que demostrar los daños. Se intenta construir un conocimiento colectivo y situado que sea una herramienta para la toma de conciencia, la visibilización de diversos problemas experimentados por las comunidades y que contribuya al empoderamiento del pueblo para transformar esa realidad.

- ¿Cómo legitimar un debate al seno de la comunidad científica mediante publicación de los resultados de las investigaciones en revistas científicas especializadas, al tiempo que se construyen, difunden y discuten los resultados en las comunidades afectadas? 
- Finalmente, e incluyendo a lo anterior, el principal desafío es descubrir cómo poner en acción el propósito transformador de esta investigación.

Bibliografía

Arancibia, F. (2012), "Las palabras y las sojas. Un enfoque desde la sociología de la ciencia y la tecnología”, Apuntes de Investigación del CECYP 22: 83-95.

Arancibia, F. (2013), "Challenging the Bioeconomy : The Dynamics of Collective Action in Argentina", Technology in Society 35: 72-92.

Arancibia, F. (2015), The Struggle to Restrict Pesticide Use: The Confluence of Social Movements and a Network of Expertise, Doctoral Dissertation, Sociology Department, State Univrsity of New York at Stony Brook, United States.

Arnulphi, C., Calvo, C., Agost, L. y J.C. Nieto (2014), "La peligrosa cercanía a los campos fumigados", Unciencia. Agencia universitaria de comunicación de la ciencia, el arte y la tecnología, Vol: Julio. Disponible: http://www.unciencia.unc.edu.ar/2014/julio/evaluan-los-efectos-de-los-agroquimicos-en-elinterior-cordobes.

Bachelard, G. (1979), Formación del Espiritu Cientifico, México: Siglo XIX.

Breilh, J. (2010), “La epidemiología crítica: una nueva forma de mirar la salud en el espacio urbano”, Salud Colectiva 6(1): 83-101.

Brown, P. (1987), "Popular Epidemiology: Community Response to Toxic Waste-Induced Disease", Science, Technology E Human Values 12(3): 78-85.

Brown, P. y E. Mikkelsen (1990), No Safe Place: Toxic Waste, Leukemia and Community Action, California: University of California Press.

Collins, H.M. y R. Evans (2002), "The Third Wave of Science Studies: Studies of Expertise and Experience", Social Studies of Science 32(2): 235-296.

CONICET (2009), "Evaluación de la información científica vinculada al glifosato en su incidencia sobre la salud humana y el ambiente", Comisión Nacional de Investigación en Agroquimicos, Consejo Científico Interdisciplinario.

FAO (2000), "Declaración de la FAO sobre Biotecnología. Biotecnologías Agrícolas", http://www.fao.org/biotech/ fao-statement-on-biotechnology/es/. Accesible al 1 de octubre de 2011.

FAO (2009), "World Summit on Food Security. Draft Declaration of the World Summit on Food Security", http://www.fao.org/fileadmin/templates/wsfs/Summit/Docs/Final_Declaration/WSFS09_Declaration.pdf. Accesible al 01 de enero de 2010.

Folguera, G., Carrizo, E. y A. Massarini (2013), "Análisis de los aspectos epistemológicos y sociales presentes en el discurso tecno-científico referido al cultivo de organismos genéticamente modificados (OGM) en la Argentina", CTS-Revista Iberoamericana de Ciencia, Tecnología y Sociedad 25(9): 91-119.

Fox Keller, E. (1995), Lenguaje y vida. Metáforas de la biología en el siglo XX, Buenos Aires: Editorial Manantial.

Giarraca, N. y M. Teubal (eds.) (2005), El campo argentino en la encrucijada, Buenos Aires: Editorial Alianza.

Giarraca, N. (2006), "Territorios en disputa: los bienes naturales en el centro de la escena”, Realidad Económica 217: 51-68.

Grupo Madres de Ituzaingó (2005), "Agroquímicos: genocidio encubierto en barrio Ituzaingó de Córdoba, Argentina”, http://www.rapaluruguay.org/agrotoxicos/Prensa/accidente_Ituzaingo.html.

Grupo de Reflexión Rural (2009), "Pueblos fumigados. Informe sobre la problemática del uso de plaguicidas en las principales provincias sojeras de la Argentina", http://www.rapaluruguay.org/agrotoxicos/Prensa/ Pueblos_Fumigados__GRR.pdf.

Habermas, J. (1970), Toward a Rational Society: Student Protest, Science and Politics, Boston: Beacon Press.

Haraway, D. (1991), Ciencia, cyborgs y mujeres: la reinvención de la naturaleza, Madrid: Ediciones Cátedra. 
Harding, S. (1995), “'Strong Objectivity': A Response to the New Objectivity Question”, Synthese 104(3): 331-349.

Hottois, G. (1999), El paradigma bioético. Una ética para la tecnociencia, Barcelona: Editorial Anthropos.

IARC Working Group (2015), “Glyphosate”, IARC 12: 1-92.

Kitcher, P. (2001), Science, Truth and Democracy, New York: Oxford University Press.

Miller, S. (2001), "Public Understanding of Science at the Crossroads", Public Understanding of Science 10: 115-120.

Levy-Leblond, J. (2003), "Una cultura sin cultura. Reflexiones críticas sobre la cultura científica", CTS-Revista Iberoamericana de Ciencia, Tecnología y Sociedad 1(1): 139-151.

Lewenstein, B.V. (2002), "A Decade of Public Understanding", Public Understanding of Science 11: 1-4.

Marcuse, H. (1968), El hombre unidimensional. Ensayo sobre la ideología de la sociedad industrial avanzada, México: Joaquín Mortiz.

Massarini, A. y A. Schnek (2015), Ciencia entre todxs. Tecnociencia en contexto social: una propuesta de enseñanza, Buenos Aires: Editorial Paidós.

Moore, K., Kleinman, D.L., Hess, D. y S. Frickel (2011), "Science and Neoliberal Globalization: A Political Sociological Approach", Theory and Society 40(5): 505-532.

Morin, E. (1981), El método, Tomo I, La naturaleza de la naturaleza, Madrid: Editorial Cátedra.

Niiniluoto, I. (1997), “Ciencia frente a Tecnología: ¿Diferencia o Identidad?”, Arbor 620: 285-299.

OECD (2009), "The Bioeconomy to 2030: Designing a Policy Agenda”, OECD, International Futures Project, Paris. http://www.oecd.org/futures/long-termtechnologicalsocietalchallenges/42837897.pdf. Accesible al 1 de Agosto de 2011.

Pavone, V. (2012), "Ciencia, Neoliberalismo y Bioeconomía", CTS-Revista Iberoamericana de Ciencia, Tecnología y Sociedad 21(7): 1-15.

Pigluicci, M. (2008), “The Borderlands Between Science and Philosophy: An Introduction”, The Quarterly Review of Biology 3(1): 7-15.

Rodríguez Alcázar, F. J. (2009), “Ciencia, tecnología y sociedad en el mundo contemporáneo”, Archivos Filosóficos del Sur 1: 107-139.

Svampa, M. (2011), "Modelo de desarrollo y cuestión ambiental en América Latina: categorías y escenarios en disputa" en Wanderley, F. (ed.), El desarrollo en cuestión. Reflexiones desde América Latina, La Paz: CIDES/ Oxfam/Plural, pp. 414-441.

Svampa, M. (2012a), "Extractivismo neodesarrollista y movimientos sociales: ¿Un giro ecoterritorial hacia nuevas alternativas?", en AA.VV. del Grupo Permanente de Trabajo sobre Alternativas al Desarrollo (eds.), Más allá del desarrollo, Quito: Fundación Rosa Luxemburgo, pp.185-218.

Svampa, M. (2012b), "Pensar el desarrollo desde América Latina", en Massuh, G. (ed.), Renunciar al bien común. Extractivismo y (pos)desarrollo en América Latina, Buenos Aires: Mardulce, pp. 17-58.

Teubal, M., Domínguez, D. y H. Sabatino (2005), "Transformaciones agrarias en la Argentina. Agricultura industrial y sistema alimentario", en Giarraca, N. y M. Teubal (eds.), El campo argentino en la encrucijada. Estrategias y resistencias sociales, ecos en la ciudad, Buenos Aires: Alianza, pp. 193-221.

Teubal, M. y N. Giarracca (2005), “Introducción: los debates acerca del campo”, en Giarraca, N. y M. Teubal (eds.), El campo argentino en la encrucijada. Estrategias y resistencias sociales, ecos en la ciudad, Buenos Aires: Alianza, pp. 19-34.

Teubal, M. (2008), "Soja y agronegocios en la Argentina: la crisis del modelo", Labvoratorio 10(22): 5-7.

UCCSNAL (2015), "Documento fundacional de la Unión de Científicos Comprometidos con la Sociedad y la Naturaleza en América Latina", http://uccsnal.org/?p=18.

Verzeñassi, D. (2016), Recordar, un ejercicio saludable. Memorias del 1er Congreso Latinoamericano de Salud Socioambiental, Buenos Aires: Editorial El Colectivo.

World Health Organization (2012), "20 questions on genetically modified foods", Biotechnology (GM foods) Publications, http://www.who.int/foodsafety/publications/biotech/20questions/en/index.html. Accesible al 1 de agosto de 2012. 
Wynne, B. (1980), “Risk, Technology and Trust: On the Social Treatment of Uncertainty", en Conrad, J. (ed.), Society, Technology and Risk, London: Arnold, pp. 83-117.

Wynne, B. (1989), "Frameworks of Rationality in Risk Management: Towards the Testing of Naïve Sociology", en Brown, J. (ed.), Environmental Threats, London: Frances Pinter, pp. 93-110.

Wynne, B. (2001), "Creating Public Alienation: Expert Cultures of Risk and Ethics on GMOs", Science as Culture 10(4): 445-481. 